$\underline{\text { Preprint typeset in JHEP style - HYPER VERSION }}$

hep-th/0507262

\title{
Gravitational duality near de Sitter space
}

\author{
Bernard Julia*, Jérôme Levie and Sébastien Ray \\ LPT, 24 rue Lhomond, 75231 Paris CEDEX 05, FRANCE \\ $E$ : bjulia levie sray AT lpt.ens.fr
}

\begin{abstract}
Nonlinear gravitational instantons " $\Lambda$-instantons" are defined here for any given value $\Lambda$ of the cosmological constant. A multiple of the Euler characteristic appears as an upper bound for the four dimensional de Sitter action and as a lower bound for a family of quadratic actions. Our new self-duality equation for the MacDowell Mansouri tensor implies the full nonlinear Einstein's equations for a given value of the cosmological constant. The de Sitter action itself is found to be equivalent to a simple and natural quadratic action. In this paper we also describe explicitly the reparameterization and duality invariances of gravity (in 4 dimensions) linearized about de Sitter space. A noncovariant doubling of the fields using the Hamiltonian formalism leads to first order time evolution with manifest duality symmetry. As a special case we recover the linear flat space result of Henneaux and Teitelboim by a smooth limiting process.
\end{abstract}

KEYwords: Duality, General relativity, de Sitter, instantons, prepotentials.

\footnotetext{
*Laboratoire de Physique théorique UMR 8549 ENS/CNRS.
} 


\section{Contents}

1. Introduction 1

2. Gravity equations with a cosmological constant 2

3. $\Lambda$-instantons 3

4. Equations near de Sitter geometry and linearized hamiltonian action

4.1 Linearization 5

4.2 Coordinate reparameterization invariances 6

5. Resolution of the constraints 6

5.1 Resolution of constraints $C_{2}^{i}$

5.2 Resolution of constraint $C_{1}$

5.3 Gauge transformation rules and preinvariances of the prepotentials

6. Duality rotations on the prepotentials 8

6.1 Physical fields on shell 8

6.2 Duality and gauge invariance 9

7. Invariance of the action 10

7.1 Action in terms of prepotentials 10

7.2 Manifestly dual expression 11

8. Conclusion 12

\section{Introduction}

We uncover here two new properties of General Relativity. The existence of duality symmetries which preserve the equations of motion and sometimes the action are important features of a theory as they frequently allow us to relate a strong coupling regime to a perturbative one which is more amenable to computation. In a linearized theory around flat space it is generally true that one can (Hodge) dualize differential form fields to get similar and sometimes even identical equations after dualization. It is much more exceptional to have nonlinear duality symmetries [1]. Their applications from Statistical mechanics to Electromagnetism or String theory are well known. We shall present a step towards nonlinear duality symmetries, namely the existence of a duality rotation group for linearized gravity around de Sitter space rather than around flat space. We recall the surprising symmetry between General Relativity and its dual theory in the linear approximation around 
Minkowski space [2]. Now in the quest for duality symmetries one may consider starting by considering self-dual solutions. In fact we shall present a new instanton equation for gravity at the full nonlinear level as a motivation for our duality analysis, the same deformation of the Riemannian curvature tensor will appear there as in the duality study.

The first result we discuss is that one can define a one parameter family of nonlinear gravitational instantons parameterized precisely by the value of the cosmological constant. Before defining this new self-duality (instanton) condition corresponding to any given value of the cosmological constant we shall review in section 3 the three previously known types of gravitational instantons. The first two types correspond respectively to special Ricci flat solutions or Einstein spaces for arbitrary values of the cosmological constant. The third type is slightly different, implying only the self-duality of the Weyl (conformal) tensor rather than that of the full Riemann curvature tensor. Our new " $\Lambda$ instantons" reduce to the first type of (ordinary) instantons when one sets the cosmological constant $\Lambda$ to zero.

To obtain the second result of this paper we shall generalize the ordinary gravitational instantons and verify the duality invariance of the linearization of General Relativity around de Sitter space. This boosts our confidence in good duality properties of gravity at the nonlinear level without assuming any isometry [3]. In section 5 we introduce prepotentials and in section 6 their duality transformation laws. Some of these correspond to diffeomorphisms whereas others are (pre)gauge symmetries. Finally section 7 is devoted to check the invariance of the action.

\section{Gravity equations with a cosmological constant}

Let us consider four dimensional spacetime with a metric either of Lorentzian signature $(-,+,+,+)$ or of euclidean (or $(2+, 2-))$ signature for the existence of instantons. Our main conventions are those of the textbook by Hawking and Ellis. The cosmological constant is denoted by $\Lambda$ and we focus on the nonvanishing case. For definiteness the computations starting with section 4 will actually be done for a positive value of $\Lambda$ and Lorentzian signature. The usual equations for the Einstein tensor read

$$
G_{\mu \nu}=-\Lambda g_{\mu \nu}
$$

Flat space can be seen either as a gravitational instanton or anti-instanton. There are (anti)-self-dual tensors in (euclidean) de Sitter space as well as in the flat space case, however they are not the chiral parts of the Riemann curvature tensor, they are rather the chiral parts of the Weyl tensor $C_{\mu \nu \rho \sigma}$ which vanishes there. Let us recall its definition:

$$
C_{\mu \nu \rho \sigma} \equiv R_{\mu \nu \rho \sigma}+\frac{1}{2}\left(g_{\mu \sigma} R_{\rho \nu}-g_{\mu \rho} R_{\sigma \nu}+g_{\nu \rho} R_{\sigma \mu}-g_{\nu \sigma} R_{\rho \mu}\right)+\frac{1}{6}\left(g_{\mu \rho} g_{\nu \sigma}-g_{\mu \sigma} g_{\nu \rho}\right) R
$$

Actually on shell the Weyl tensor $C_{\mu \nu \rho \sigma}$ is equal to a new tensor $Z_{\mu \nu \rho \sigma}$ defined as follows:

$$
Z_{\mu \nu \rho \sigma} \equiv R_{\mu \nu \rho \sigma}-\frac{\Lambda}{3}\left(g_{\mu \rho} g_{\nu \sigma}-g_{\mu \sigma} g_{\nu \rho}\right)
$$

How does one discover this tensor $Z_{\mu \nu \rho \sigma}$ ? One way to find it is to require the form of the equations of motion and of the torsion Bianchi identity to be the same. This is well 
known in the absence of cosmological constant and it turns out that the only required modification to accommodate a nonvanishing $\Lambda$ is precisely to replace $R_{\mu \nu \rho \sigma}$ by $Z_{\mu \nu \rho \sigma}$. Symmetry properties and even topological inequalities can be extended from one to the other to a very large extent. In fact $Z_{\mu \nu \rho \sigma}$ obeys simple translation and also rotation Bianchi identities

$$
\begin{aligned}
Z_{\mu[\nu \rho \sigma]} & \equiv 0 \\
\nabla_{[\mu} Z_{\nu \rho] \sigma \tau} & \equiv 0
\end{aligned}
$$

(there is no torsion.)

The equally beautiful equations of motion read:

$$
Z_{\mu \nu} \equiv Z_{\mu \rho \nu}^{\rho}=0 .
$$

(Note that this would be an identity for the Weyl tensor). Let us remark that the dual tensor:

$$
\tilde{Z}_{\mu \nu \rho \sigma} \equiv \frac{1}{2} \epsilon_{\mu \nu \alpha \beta} Z_{\rho \sigma}^{\alpha \beta}
$$

obeys the same equations but with reverse meaning. The torsion Bianchi identity becomes the dual equation of motion $\tilde{Z}_{\mu \nu}=0$, and the equation of motion has the form of the first Bianchi identity $\tilde{Z}_{\mu[\nu \rho \sigma]}=0$ for the dual $\tilde{Z}_{\mu \nu \rho \sigma}$ tensor.

\section{3. $\Lambda$-instantons}

From the above remarks it is natural to define the (anti)- $\Lambda$-instantons with given value $\Lambda$ of the cosmological constant by the equation

$$
\tilde{Z}_{\mu \nu \rho \sigma}= \pm Z_{\mu \nu \rho \sigma}
$$

They automatically obey the second order Einstein equations for that particular value of $\Lambda$. We recall that similar equations define the usual gravitational (anti)-instantons. For Ricci flat metrics we further require that

$$
* R_{\mu \nu \rho \sigma}= \pm R_{\mu \nu \rho \sigma}
$$

where the star means Hodge dualisation on the second pair of indices. These are nothing but $(\Lambda=0)$-instantons.

For Einstein spaces i.e. for an a priori undetermined value of the cosmological constant, one may speak of "cosmoinstantons" because the Einstein equations can be equivalently rewritten

$$
* \tilde{R}_{\mu \nu \rho \sigma}=R_{\mu \nu \rho \sigma}
$$

where the tilde means dualization on the first pair of indices as above [4, 匇. However we could reject the name instanton in that cas because the equations remain of second order. There is a Hitchin-Bogomolny-Prasad-Sommerfield type inequality bounding below the quadratic Yang action by multiples respectively of the Pontryagin and Euler invariants. Another similar inequality has been introduced in 8] in relation with the conformal 
instantons ie those instantons for which the Weyl tensor is self-dual (say on its first pair of indices). We may now notice that the ordinary (anti)-instantons and more generally (anti)- $\Lambda$-instantons are instances of cosmoinstantons and are also (anti)-conformal instantons.

$\Lambda$-instantons saturate analogously a BPS condition obtained by replacing the curvature tensor $R$ by the new tensor $Z$ in the Yang action. The corresponding action $S_{\Lambda}^{(2)} \equiv$ $\int \operatorname{tr}(Z . Z) d^{4} x$ is bounded below by $J_{\Lambda}(g)$ where we defined $J_{\Lambda}(g) \equiv \int \operatorname{tr}(Z \tilde{Z}) d^{4} x$. The right hand side lower bound of the BPS condition is still proportional to the signature invariant.

However the double self-duality equations $\tilde{Z}_{\mu \nu \rho \sigma}=* Z_{\mu \nu \rho \sigma}$ does not seem a priori interesting for our purpose as it only implies Einstein's equations for some arbitrary value of the cosmological constant, exactly as in the case of ordinary duality $\tilde{R}_{\mu \nu \rho \sigma}=* R_{\mu \nu \rho \sigma}$. Note however that this "double self-duality" leads to a BPS bound that is not a topological invariant, we find:

$$
I_{\Lambda}(g) \equiv \int \operatorname{tr}(\tilde{Z} \tilde{Z}) d^{4} x=32 \pi^{2} \chi_{E u l e r}-4 / 3 \Lambda S_{\Lambda}
$$

where

$$
S_{\Lambda}=\int[R-2 \Lambda] \sqrt{g} d^{4} x
$$

is the Hilbert-Einstein-de Sitter action under consideration. This simple computation shows that the quadratic action $I_{\Lambda}(g)$ is equivalent to the a priori better quantum behaved de Sitter action.

Equation 3.4 is a rederivation of the famous MacDowell Mansouri formula [5] for de Sitter action. Again we have

$$
S_{\Lambda}^{(2)} \geq I_{\Lambda}(g)
$$

The bound given above is not anymore the Euler topological invariant, it differs from it by a multiple of $S_{\Lambda}$. The expression $I_{\Lambda}(g)$ is non negative hence

$$
\Lambda S_{\Lambda} \leq 24 \pi^{2} \chi_{\text {Euler }}
$$

We can rewrite the BPS inequality as

$$
\Lambda S_{\Lambda} \geq 24 \pi^{2} \chi_{E u l e r}-3 / 4 \int \operatorname{tr}(Z . Z) d^{4} x
$$

or more suggestively

$$
\Lambda S_{\Lambda}+3 / 4 S_{\Lambda}^{(2)} \geq 24 \pi^{2} \chi_{\text {Euler }}
$$

We find that exactly for cosmoinstantons we have equality whatever the value of the cosmological constant. We hope to return to this in the future.

\section{Equations near de Sitter geometry and linearized hamiltonian action}

At the linear level one may replace the covariant derivative $\nabla$ by $\partial$ in the rotation Bianchi identity. This identity is the same as its dual because there is no torsion (even off shell) and 
hence $Z_{\mu \nu \rho \sigma}$ is symmetric under the exchange of its antisymmetric pairs of indices. Let us rephrase the result we have obtained: if one is careful to replace the Riemann tensor $R_{\mu \nu \rho \sigma}$ by the $Z_{\mu \nu \rho \sigma}$ tensor which vanishes on de Sitter shell, the linearized equations exhibit selfduality near de Sitter space. This extends the result of Henneaux and Teitelboim [2] to the case of a non-vanishing cosmological constant. The idea of considering this generalization was also proposed in [6] and was motivated by supergravity. We will now analyze in detail this symmetry and define dual potentials as well as prepotentials and their duals to exhibit a continuous (one parameter) symmetry group of the action.

We shall use an $\mathrm{SO}(3)$ covariant choice of coordinates covering one half of de Sitter spacetime, the so-called planar coordinates: the $d S_{4}$ metric can be written

$$
d s^{2}=-d t^{2}+f^{2}(t) \delta_{i j} d x^{i} d x^{j}
$$

Our latin indices refer to spatial directions whereas greek ones are four dimensional. Up to a constant we have $f(t)=\exp (t \sqrt{\Lambda / 3})=\exp (K t)$, where it is convenient to define the inverse de Sitter radius $K \equiv \sqrt{\Lambda / 3}$. Following [9] we can write the general gravity action in hamiltonian or $3+1$ form namely

$S=\int d^{4} x\left[\pi^{i j} \dot{g}_{i j}+N g_{3}^{1 / 2}\left(R^{(3)}-2 \Lambda\right)+N g_{3}^{-1 / 2}\left(\frac{1}{2} \pi^{2}-g_{i k} g_{j l} \pi^{i j} \pi^{k l}\right)+2 N_{i}\left(\partial_{j} \pi^{i j}+\Gamma_{j k}^{(3) i} \pi^{j k}\right)\right]$

with

$$
\begin{array}{r}
N=\left(-g^{00}\right)^{-1 / 2}, \quad N_{i}=g_{0 i}, \\
g_{3}=\operatorname{det}\left(g_{i j}\right), \quad \pi=g_{i j} \pi^{i j} .
\end{array}
$$

Here $g_{i j}$ is the spatial metric, $\pi^{i j}$ its conjugate momentum (density), $R^{(3)}$ is the 3D scalar curvature and $\Gamma^{(3)}$ the $3 \mathrm{D}$ Levi-Civita connection associated to $g_{i j}$.

\subsection{Linearization}

Let us now expand around de Sitter background.

$$
\begin{gathered}
g_{i j}=f^{2}(t) \delta_{i j}+h_{i j}, \quad \pi^{i j}=\bar{\pi}^{i j}+p^{i j}, \\
N=1+n, \quad N_{i}=n_{i} .
\end{gathered}
$$

We find the background value of the momenta:

$$
\bar{\pi}^{i j}=-2 K f \delta^{i j}
$$

Now to second order the various terms read (using the flat euclidean metric to raise and lower the indices and to take traces (for instance $h$ and $p$ )): 


$$
\begin{aligned}
g_{3}= & f^{6}\left(1+f^{-2} h+\frac{1}{2} f^{-4}\left(h^{2}-h^{i j} h_{i j}\right)\right) \\
-\sqrt{g_{3}} R^{(3)}= & f^{-1}\left(\Delta h-\partial_{i} \partial_{j} h^{i j}\right)+f^{-3}\left[\frac{1}{2} h\left(\Delta h-\partial_{i} \partial_{j} h^{i j}\right)\right. \\
& -h^{i j}\left(\partial_{i} \partial_{j} h+\Delta h_{i j}-2 \partial_{i} \partial^{k} h_{j k}\right)-\frac{3}{4} \partial^{i} h^{j k} \partial_{i} h_{j k}+\frac{1}{2} \partial^{i} h^{j k} \partial_{j} h_{i k} \\
& \left.-\partial^{i} h_{i j} \partial^{j} h+\partial^{i} h_{i j} \partial_{k} h^{j k}+\frac{1}{4} \partial_{i} h \partial^{i} h\right] \\
\frac{1}{2} \pi^{2}-g_{i k} g_{j l} \pi^{i j} \pi^{k l}= & -f^{2}\left(f^{2} p^{i j} p_{i j}-2 K f p^{i j} h_{i j}+4 K^{2} h^{i j} h_{i j}\right) \\
& +\frac{f^{2}}{2}(f p-2 K h)^{2}-2 K f^{4}(f p-2 K h)+6 K^{2} f^{6}
\end{aligned}
$$

It follows that the quadratic action near de Sitter space is

$$
S=\int d x^{4}\left(p^{i j} \dot{h}_{i j}-H-n C_{1}-n_{i} C_{2}^{i}\right)
$$

with :

$$
\begin{aligned}
H= & f^{-3}\left(\frac{1}{4} \partial^{i} h^{j k} \partial_{i} h_{j k}-\frac{1}{4} \partial^{i} h \partial_{i} h+\frac{1}{2} \partial^{i} h \partial^{j} h_{i j}-\frac{1}{2} \partial_{i} h^{i j} \partial^{k} h_{j k}\right) \\
& +f^{-1}\left(f^{2} p^{i j} p_{i j}-2 K f p^{i j} h_{i j}+K^{2} h_{i j} h^{i j}\right)-\frac{1}{2} p(f p-2 K h), \\
C_{1}= & -f^{-1}\left(\partial^{i} \partial^{j} h_{i j}-\Delta h\right)+2 f K(f p+K h), \\
C_{2}^{i}= & -2 \partial_{j} p^{i j}+2 f^{-1} K\left(2 \partial_{j} h^{i j}-\partial^{i} h\right) .
\end{aligned}
$$

Extremization of the action leads to the vanishing of the constraints $C_{1}$ and $C_{2}^{i}$.

\subsection{Coordinate reparameterization invariances}

Spatial diffeomorphisms transform $h_{i j}$ into $h_{i j}+\partial_{i} \xi_{j}+\partial_{j} \xi_{i}$. On the other hand time dependent coordinate transformations are as usual generated by the primary constraints

which have been solved here. The space dependent time reparameterization invariance must be generated by the hamiltonian constraint. The scalar and vector constraints generate canonically the $\xi$ and $\xi_{i}$ gauge variations (reparameterizations) of an observable $\phi$, $\delta \phi=\left\{\xi C_{1}-\xi_{i} C_{2}^{i}, \phi\right\}$, and in particular :

$$
\left\{\begin{array}{l}
\delta h_{i j}=\partial_{i} \xi_{j}+\partial_{j} \xi_{i}-2 K f^{2} \delta_{i j} \xi \\
\delta p_{i j}=f^{-1}\left(-\partial_{i} \partial_{j} \xi+\delta_{i j} \Delta \xi\right)+2 K f^{-1}\left(\partial_{i} \xi_{j}+\partial_{j} \xi_{i}-\delta_{i j} \partial_{k} \xi^{k}\right)+2 K^{2} f \delta_{i j} \xi
\end{array}\right.
$$

\section{Resolution of the constraints}

\subsection{Resolution of constraints $C_{2}^{i}$}

The vector constraint reads

$$
\partial_{j}\left[p^{i j}-K f^{-1}\left(2 h^{i j}-\delta^{i j} h\right)\right]=0
$$


equivalently $\partial_{j} a^{i j}=0$ where $a^{i j} \equiv p^{i j}-K f^{-1}\left(2 h^{i j}-\delta^{i j} h\right)$ is a symmetric tensor. Hence

$$
a^{i j}=f^{-1} \epsilon^{i k l} \epsilon^{j m n} \partial_{k} \partial_{m} P_{l n}
$$

where the prepotential $P_{i j}$ is defined up to an ambiguity $\delta P_{i j}=\partial_{i} \alpha_{j}+\partial_{j} \alpha_{i}$. The factor $f$ is introduced here for later convenience as it simplifies the equations and depends only on time. Finally we obtain

$$
f p^{i j}=\delta^{i j}\left(\Delta P-\partial^{k} \partial^{l} P_{k l}\right)+\partial^{i} \partial^{k} P^{j}{ }_{k}+\partial^{j} \partial^{k} P^{i}{ }_{k}-\partial^{i} \partial^{j} P-\Delta P^{i j}+K\left(2 h^{i j}-\delta^{i j} h\right) .
$$

\subsection{Resolution of constraint $C_{1}$}

We deduce from above that $f p=\Delta P-\partial^{i} \partial^{j} P_{i j}-K h$. The scalar constraint can be rewritten:

$$
\partial_{i} \partial_{j} b^{i j}-\Delta b=0
$$

where $b_{i j}=h_{i j}+2 K f^{2} P_{i j}$. Its general solution is

$$
b_{i j}=f\left(\epsilon_{i k l} \partial^{k} \Phi_{j}^{l}+\epsilon_{j k l} \partial^{k} \Phi_{i}^{l}\right)+\partial_{i} u_{j}+\partial_{j} u_{i},
$$

with the second prepotential $\Phi$ a symmetric tensor defined up to the arbitrariness $\delta \Phi_{i j}=$ $\partial_{i} \beta_{j}+\partial_{j} \beta_{i}+\delta_{i j} \eta$, provided one varies the third prepotential as follows $\delta u_{i}=-\epsilon_{i j k} \partial^{j} \beta^{k}$. Again a factor $f$ has been introduced for future simplifications. It follows that

$$
h_{i j}=f\left(\epsilon_{i k l} \partial^{k} \Phi_{j}^{l}+\epsilon_{j k l} \partial^{k} \Phi_{i}^{l}\right) \partial_{i} u_{j}+\partial_{j} u_{i}-2 K f^{2} P_{i j}
$$

\subsection{Gauge transformation rules and preinvariances of the prepotentials}

The coordinate reparameterization invariance must be implemented on the prepotentials we just defined in (5.3) and (5.6) by some gauge transformation laws $(\delta P, \delta \Phi, \delta u)$ up to possible preinvariances that do not affect the original gravity fields and momenta. We obtain first the equation:

$$
\epsilon^{i k l} \epsilon^{j m n} \partial_{k} \partial_{m} \delta P_{l n}=\left(-\partial^{i} \partial^{j} \xi+\delta^{i j} \Delta \xi\right)
$$

whose general solution is:

$$
\delta P_{i j}=\delta_{i j} \xi+\partial_{i} \alpha_{j}+\partial_{j} \alpha_{i}
$$

We now use this to determine $\delta \Phi$ :

$$
f\left(\epsilon_{i k l} \partial^{k} \delta \Phi_{j}^{l}+\epsilon_{j k l} \partial^{k} \delta \Phi_{i}^{l}\right)=\partial_{i}\left(\xi_{j}-u_{j}+2 K f^{2} \alpha_{j}\right)+\partial_{j}\left(\xi_{i}-u_{i}+2 K f^{2} \alpha_{i}\right) .
$$

Its general solution is given by :

$$
\begin{aligned}
\delta \Phi_{i j} & =\partial_{i} \beta_{j}+\partial_{j} \beta_{i}+\delta_{i j} \eta \\
\delta u_{i} & =\xi_{i}-f \epsilon_{i j k} \partial^{j} \beta^{k}+2 K f^{2} \alpha_{i}
\end{aligned}
$$

Preinvariances are parameterized by $\alpha_{i}, \beta_{i}$ and $\eta$, they leave invariant $h_{i j}$ and $p_{i j}$, whereas gauge invariances -parameterized by $\xi_{i}$ and $\xi$-act on these fields but leave the action invariant. 


\section{Duality rotations on the prepotentials}

The benefit of having solved the constraints is that the lapse and shift (Lagrange multipliers $n$ and $n_{i}$ ) drop out. The new lagrangian is $L=p^{i j} \dot{h}_{i j}-H$. We will need to reexpress it in terms of the prepotentials but let us start with the computation of the "electric" and "magnetic" gravitational fields (on shell). Namely let us define the $3 \mathrm{D}$ tensors $\mathcal{E}_{i j} \equiv Z_{i 0 j 0}$

and $\mathcal{B}_{i j} \equiv \tilde{Z}_{i 0 j 0}$. In the absence of torsion and on shell the remaining components of the Riemannian curvature are expressible in terms of these.

\subsection{Physical fields on shell}

In the linear approximation one finds for $\mathcal{E}$

$$
\begin{aligned}
-\mathcal{E}_{i j}= & \frac{1}{2}\left(\partial_{i} \partial_{j} h_{00}+\partial_{0} \partial_{0} h_{i j}-\partial_{i} \partial_{0} h_{j 0}-\partial_{j} \partial_{0} h_{i 0}\right) \\
& -K \partial_{0}\left(h_{i j}-\frac{1}{2} f^{2} h_{00} \delta_{i j}\right)
\end{aligned}
$$

The hamiltonian equations of motion read

$$
\begin{aligned}
\dot{h}_{i j}= & 2 f p_{i j}-f p \delta_{i j}+\left(\partial_{i} n_{j}+\partial_{j} n_{i}\right)+K\left(-2 h_{i j}+h \delta_{i j}+2 f^{2} n \delta_{i j}\right) \\
-\dot{p}_{i j}= & \frac{1}{2} f^{-3}\left(-\Delta h_{i j}-\partial_{i} \partial_{j} h+\partial_{i} \partial^{k} h_{j k}+\partial_{j} \partial^{k} h_{i k}+\Delta h \delta_{i j}-\partial_{k} \partial_{l} h^{k l} \delta_{i j}\right) \\
& -f^{-1} \partial_{i} \partial_{j} n+f^{-1} \Delta n \delta_{i j}+2 K^{2} f^{-1} h_{i j}+2 K^{2} f n \delta_{i j} \\
& +K\left[\left(-2 p_{i j}+p \delta_{i j}\right)-2 f^{-1}\left(\partial_{i} n_{j}+\partial_{j} n_{i}\right)+2 f^{-1} \partial_{k} n^{k} \delta_{i j}\right]
\end{aligned}
$$

Using $h_{00}=-2 n$ and $h_{i 0}=n_{i}$ and the scalar constraint we may check that

$$
\begin{aligned}
-\mathcal{E}_{i j}= & \frac{1}{2} f^{-2}\left(\Delta h_{i j}+\partial_{i} \partial_{j} h-\partial_{i} \partial^{k} h_{j k}-\partial_{j} \partial^{k} h_{i k}\right) \\
& -f K\left(p_{i j}-p \delta_{i j}\right)+2 K^{2} h_{i j}-\frac{K^{2}}{2} h \delta_{i j} .
\end{aligned}
$$

In terms of prepotentials and for solutions of the equations of motion

$$
-\mathcal{E}_{i j}=\frac{1}{2} f^{-1}\left(\epsilon_{i k l} \partial_{j} \partial_{m} \partial^{k} \Phi^{l m}+\epsilon_{j k l} \partial_{i} \partial_{m} \partial^{k} \Phi^{l m}-\epsilon_{i k l} \partial_{k} \Delta \Phi_{j}^{l}-\epsilon_{j k l} \partial_{k} \Delta \Phi_{i}^{l}\right)
$$

Let us note that this expression does not depend explicitly on the cosmological constant, please observe that it depends only on $\Phi$ and that it is gauge and pregauge invariant. Also up to a rescaling it is the same expression as around flat space. This suggests that it should follow simply from the analysis of the Weyl tensor because on shell $C_{\mu \nu \rho \sigma}=Z_{\mu \nu \rho \sigma}$ and of course de Sitter is conformally flat.

Let us now compute the magnetic expression $\mathcal{B}$. By a tedious computation one may rewrite

$$
-\mathcal{B}_{i j}=\frac{1}{2} f^{-1} \epsilon_{i}{ }^{k l} R_{k l j}{ }^{0}-\frac{1}{2} f^{-1} \epsilon_{i}{ }^{k l} Z_{k l j}{ }^{m} n_{m},
$$

where $\epsilon$ is the antisymetric tensor of 3D euclidean space. To first order

$$
-R_{i j k}{ }^{0}=\partial_{0} \partial_{[i} h_{j] k}-2 K \partial_{[i} h_{j] k}+\partial_{k} \partial_{[j} n_{i]}+2 f^{2} K \delta_{k[i} \partial_{j]} n .
$$


Now to zeroth order, $Z_{k l j}{ }^{m}=0$. Again on shell one finds

$$
-\mathcal{B}_{i j}=\epsilon_{i}^{k l} \partial_{k}\left[p_{j l}-\frac{1}{2} p \delta_{j l}-2 K f^{-1}\left(h_{j l}-\frac{1}{4} h \delta_{j l}\right)\right] .
$$

Let us now check that $\mathcal{B}_{i j}$ is symmetric in its two indices by contraction with the tensor $\epsilon^{i j m}$, we find that its antisymmetric part:

$$
-\epsilon^{i j m} \mathcal{B}_{i j}=\partial_{i} p^{i m}-2 f^{-1} K \partial_{i} h^{i m}+f^{-1} K \partial^{m} h=0
$$

vanishes thanks to the vector constraint. We finally discover that

$$
-\mathcal{B}_{i j}=\frac{1}{2} \partial_{k}\left[\epsilon_{i}^{k l}\left(p_{j l}-2 K f^{-1} h_{j l}\right)+\epsilon_{j}^{k l}\left(p_{i l}-2 K f^{-1} h_{i l}\right)\right]
$$

or as a function of the superpotentials :

$$
-\mathcal{B}_{i j}=\frac{1}{2} f^{-1}\left(\epsilon_{i k l} \partial_{j} \partial_{m} \partial^{k} P^{l m}+\epsilon_{j k l} \partial_{i} \partial_{m} \partial^{k} P^{l m}-\epsilon_{i k l} \partial_{k} \Delta P_{j}^{l}-\epsilon_{j k l} \partial_{k} \Delta P_{i}^{l}\right)
$$

Now this expression is proportional to the flat space case expression of [2] which depends only on $P$ and is gauge invariant.

\subsection{Duality and gauge invariance}

The infinitesimal duality transformation rotates $\mathcal{E}$ into $\mathcal{B}: \delta \mathcal{E}=\mathcal{B}, \delta \mathcal{B}=-\mathcal{E}$. Similarly $\delta \Phi=$ $P, \delta P=-\Phi$. On the other hand if one applies a $\pi / 2$ duality rotation one obtains $\Phi^{\prime}=P$ and $P^{\prime}=-\Phi$. One may define a dual metric $h_{i j}^{\prime}$ given in terms of prime variables as in (5.6) with due replacements. As a result we have at our disposal (in this approximation) a one parameter family of metrics on 4D space provided the action of the duality transformation is non trivial. It is an important question to determine whether the 4-manifolds can be assembled into a higher dimensional structure for instance.

But let us focus on discrete duality for the time being. Is there a dual coordinate reparameterization invariance? On the face of it there is a single spacetime on which the metrics are computed. Actually in the flat space perturbative analysis of [2] the prepotential $u$ was discarded by a coordinate choice (essentially a spatially harmonic gauge if one fixes also appropriately the pregauge invariances). But we could try and preserve the coordinate reparameterization invariance and make it compatible with duality. This means probably that one should introduce a dual "spatial" coordinate reparameterization and that means in turn that one should presumably have a second set of coordinates, something string theorists might appreciate.

We may collect the set of transformation rules with dual pairs of parameters to emphasize their symmetry under duality:

$$
\begin{aligned}
\delta P_{i j} & =\delta_{i j} \xi+\partial_{i} \alpha_{j}+\partial_{j} \alpha_{i} . \\
\delta \Phi_{i j} & =\delta_{i j} \eta+\partial_{i} \beta_{j}+\partial_{j} \beta_{i} \\
\delta u_{i} & =\xi_{i}-f \epsilon_{i j k} \partial^{j} \beta^{k}+2 K f^{2} \alpha_{i}
\end{aligned}
$$


We may now ask for the fate of the gauge and pregauge invariances under duality. The absence of a dual $v_{m}$ to $u_{m}$ breaks the duality symmetry, however $u$ is pure diffeomorphism gauge and we may ignore it for the rest of this paper if we ignore $v$. Introducing primes on the parameters as above after discrete duality we get for the dual parameters:

$$
\begin{aligned}
\alpha_{i}^{\prime} & =-\beta_{i} \\
\beta_{i}^{\prime} & =\alpha_{i} \\
\xi^{\prime} & =-\eta \\
\eta^{\prime} & =\xi
\end{aligned}
$$

It maybe worthwhile to investigate space(time) doubling as suggested above, we would need

$$
\xi_{i}^{\prime}=\delta u_{i}^{\prime}+f \epsilon_{i j k} \partial^{j} \alpha^{k}+2 K f^{2} \beta_{i}
$$

\section{Invariance of the action}

We are now ready to rewrite the action in terms of $P, \Phi$ ( $u$ disappears because of coordinate reparameterization invariance).

\subsection{Action in terms of prepotentials}

We saw that after solving the constraints the lagrangian simplifies to $L=p^{i j} \dot{h}_{i j}-H$. Let us call $H_{1}$ the part of $H$ that depends on $\partial h$ and $H_{2}$ the rest of it with quadratic terms $p^{2}, p h, h^{2}$. Let us define $\mathcal{P}^{i j} \equiv f p^{i j}-K\left(2 h^{i j}-\delta^{i j} h\right)$ :

$$
f H_{2}=\mathcal{P}^{i j} \mathcal{P}_{i j}-\frac{1}{2}\left(\Delta P-\partial_{i} \partial_{j} P^{i j}\right)^{2}+2 K h_{i j} \mathcal{P}^{i j}+K^{2}\left(h^{i j} h_{i j}-\frac{1}{2} h^{2}\right)
$$

Let us now compute $p^{i j} \dot{h}_{i j}$, it is equal up to a total derivative to:

$$
f p^{i j} \dot{h}_{i j}=K^{2}\left(h^{i j} h_{i j}-\frac{1}{2} h^{2}\right)+\mathcal{P}^{i j} \dot{h}_{i j}
$$

Hence

$$
f L=\mathcal{P}^{i j} \dot{h}_{i j}-2 K \mathcal{P}^{i j} h_{i j}-\left[\mathcal{P}^{i j} \mathcal{P}_{i j}-\frac{1}{2}\left(\Delta P-\partial_{i} \partial_{j} P^{i j}\right)^{2}\right]-f H_{1}
$$

We obtain term by term up to total derivative terms again:

$$
\begin{aligned}
\mathcal{P}^{i j} \mathcal{P}_{i j}- & \frac{1}{2}\left(\Delta P-\partial_{i} \partial_{j} P^{i j}\right)^{2} \\
= & \Delta P^{i j} \Delta P_{i j}-\frac{1}{2} \Delta P \Delta P+2 \partial_{i} P^{i j} \Delta \partial^{k} P_{j k}+\Delta P \partial_{i} \partial_{j} P^{i j}+\frac{1}{2}\left(\partial_{i} \partial_{j} P^{i j}\right)^{2} \\
\mathcal{P}^{i j} \dot{h}_{i j}= & 2 f \epsilon_{i j k} \partial^{j}\left[\dot{\Phi}_{l}^{k}+K \Phi_{l}^{k}\right]\left(\partial^{l} \partial_{m} P^{m i}-\Delta P^{i l}\right)-3 K^{2} f^{2}\left(P \Delta P-P_{i j} \Delta P^{i j}\right. \\
& \left.-2 P \partial_{i} \partial_{j} P^{i j}-2 \partial_{i} P^{i j} \partial^{k} P_{j k}\right)
\end{aligned}
$$




$$
\begin{aligned}
& 2 K \mathcal{P}^{i j} h_{i j}=4 K f \epsilon_{i j k} \partial^{j} \Phi_{l}^{k}\left(\partial^{l} \partial_{m} P^{m i}-\Delta P^{i l}\right)-4 K^{2} f^{2}\left(P \Delta P-P_{i j} \Delta P^{i j}\right. \\
& \left.-2 P \partial_{i} \partial_{j} P^{i j}-2 \partial_{i} P^{i j} \partial^{k} P_{j k}\right) \\
& H_{1}=f^{-1}\left(\Delta \Phi^{i j} \Delta \Phi_{i j}-\frac{1}{2} \Delta \Phi \Delta \Phi+2 \partial_{i} \Phi^{i j} \Delta \partial^{k} \Phi_{j k}+\Delta \Phi \partial_{i} \partial_{j} \Phi^{i j}+\frac{1}{2}\left(\partial_{i} \partial_{j} \Phi^{i j}\right)^{2}\right) \\
& +K^{2} f\left(P \Delta P-P_{i j} \Delta P^{i j}-2 P \partial_{i} \partial_{j} P^{i j}-2 \partial_{i} P^{i j} \partial^{k} P_{j k}\right) \\
& -2 K \epsilon_{i j k} \partial^{j} \Phi_{l}^{k}\left(\partial^{l} \partial_{m} P^{m i}-\Delta P^{i l}\right)
\end{aligned}
$$

At last :

$$
\begin{aligned}
L= & -f^{-1}\left(\Delta \Phi^{i j} \Delta \Phi_{i j}-\frac{1}{2} \Delta \Phi \Delta \Phi+2 \partial_{i} \Phi^{i j} \Delta \partial^{k} \Phi_{j k}+\Delta \Phi \partial_{i} \partial_{j} \Phi^{i j}+\frac{1}{2}\left(\partial_{i} \partial_{j} \Phi^{i j}\right)^{2}\right) \\
& -f^{-1}\left(\Delta P^{i j} \Delta P_{i j}-\frac{1}{2} \Delta P \Delta P+2 \partial_{i} P^{i j} \Delta \partial^{k} P_{j k}+\Delta P \partial_{i} \partial_{j} P^{i j}+\frac{1}{2}\left(\partial_{i} \partial_{j} P^{i j}\right)^{2}\right) \\
& +2 \epsilon_{i j k} \partial_{l} \dot{\Phi}^{k l} \partial^{j} \partial_{m} P^{i m}-2 \epsilon_{i j k} \partial^{j} \dot{\Phi}^{k l} \Delta P^{i}{ }_{l}
\end{aligned}
$$

The first two lines are manifestly invariant under duality $\delta \Phi=P, \delta P=-\Phi$. Let us vary the penultimate term $A \equiv \epsilon_{i j k} \partial_{l} \dot{\Phi}^{k l} \partial^{j} \partial_{m} P^{i m}$ :

$$
\delta A=\epsilon_{i j k} \partial_{l} \dot{P}^{k l} \partial^{j} \partial_{m} P^{i m}-\epsilon_{i j k} \partial_{l} \dot{\Phi}^{k l} \partial^{j} \partial_{m} \Phi^{i m}
$$

Using antisymmetry in $i$ and $k$ and up to a spatial derivative term :

$$
\delta A=\frac{1}{2} \partial_{0}\left(\epsilon_{i j k} \partial_{l} P^{k l} \partial^{j} \partial_{m} P^{i m}-\epsilon_{i j k} \partial_{l} \Phi^{k l} \partial^{j} \partial_{m} \Phi^{i m}\right)
$$

The last term in the lagrangian is also a time derivative and the full action is therefore duality invariant.

\subsection{Manifestly dual expression}

We have extended the noncovariant action of [2] to the de Sitter background with minor modifications by powers of $f$. We leave it for another project to render the whole presentation Lorentz covariant. But let us conclude here with a manifestly dually symmetric action. Defining the doubled prepotentials

$$
\left(A_{a}^{m n}\right)_{a=1,2}=\left(P^{m n}, \Phi^{m n}\right),
$$

we get

$$
\begin{aligned}
L= & \epsilon^{a b} \epsilon^{m n p}\left(\partial^{q} \partial_{n} \partial_{r} A_{a q p}-\Delta \partial_{n} A_{a r p}\right) \dot{A}_{b m}^{r} \\
& -\delta^{a b} f^{-1}\left\{\Delta A_{a m n} \Delta A_{b}^{m n}+\frac{1}{2} \partial^{m} \partial^{n} A_{a m n} \partial^{p} \partial^{q} A_{b p q}+\partial^{m} \partial^{n} A_{a m n} \Delta A_{b}\right. \\
& \left.-2 \partial_{m} \partial_{n} A_{a}^{m p} \partial^{n} \partial^{q} A_{b p q}-\frac{1}{2} \Delta A_{a} \Delta A_{b}\right\} .
\end{aligned}
$$


The Noether charge that generates the duality rotation is strictly identical to that of [2]. One may extend duality also outside the constraint surface by the same general procedure too.

\section{Conclusion}

Let us hope that the $\Lambda$-instantons have more uses - they deserve closer investigation -, and that the $Z_{\mu \nu \rho \sigma}$ tensor finds its truly geometrical interpretation. We have shown that duality invariance of quasi-flat gravity can be extended to the quasi-de Sitter case, which certainly is a large perturbation away. It is an encouraging indication that the duality features of $4 \mathrm{D}$ General Relativity may be simpler than might be expected from the dualization procedure of Eddington and Schroedinger [10, 1].

\section{Acknowledgments}

We benefited from discussions with Y. Dolivet, M. Henneaux and J. Iliopoulos and U. Lindstrom.

\section{References}

[1] B. Julia, M. Perry and I. Dolgachev work in progress announced at the Lebedev International conference on Theoretical Physics, Moscow April 15, 2005.

[2] M. Henneaux and C. Teitelboim, Phys.Rev. D71 (2005) 024018.

[3] In his talks on the subject B.J. usually emphasizes the challenge to "understand" a geometrical reason for those hidden dualities that do not come obviously from dimensional reduction. In the Cargèse lectures hep-th/9805083 he argued for the fusion of the spacetime symmetries and the dualities inside a group that is independent on the dimension of spacetime under consideration. The reduction of this group into the above two factors is done differently in each dimension. This is one example of action of the duality groups above their observed dimension.

[4] A.A. Belavin and D.E. Burlankov, Phys.Lett.58A (1976) 7. See also M.F. Atiyah, N.J. Hitchin and I.M. Singer, Proc.Roy.Soc.London A362 (1978) 425.

[5] S.W. MacDowell and F. Mansouri, Phys.Rev.Lett. 38 (1977) 739, erratum ibid. 38 (1977) 1376.

[6] S. Deser and D. Seminara, Phys.Lett.B607 (2005) 317.

[7] T. Eguchi and P.G.O. Freund, Phys.Rev.Lett.37 (1976) 1251.

[8] S. Deser and R. Nepomechie, Phys.Lett.97A (1983) 329.

[9] P.A.M. Dirac, Proc.Roy.Soc.A246 (1958) 333, R. Arnowitt, S. Deser and C.W. Misner (1962) reproduced in the archive gr-qc/0405109 and L. Abbott and S. Deser Nuc.Phys.B195 (1982) 76.

[10] E. Schroedinger, Proc.Roy.Ir.Ac. 51 (1947) 163. 